\title{
„Du bist ein Symbol, Mensch! Du bist ein echtes Symbol!“‘
}

\section{Metapragmatische Positionierungen im Diskurs zur deutschen Revolution 1918/19}

\begin{abstract}
Der Beitrag geht von der Überlegung aus, dass Mehrsprachigkeit nicht essentiell fixiert werden kann, sondern an von Akteuren wahrgenommene Differenzen gebunden ist. Dieser Annahme folgend entwickelt er im ersten Teil, ausgehend vom Konzept der sozialen Registrierung, ein Theoriemodell, mit dem die Koppelung unterschiedlicher Formen des Sprechens an verschiedene soziale Bedeutungen (Akteure, Praktiken, Kontexte) erfasst werden kann. Im Rückgriff auf dieses Modell analysiert der zweite Teil das Vorkommen verschiedener Formen des Sprechens in Alfred Döblins November 1918. Das Evozieren von Sprechweisen, die im Roman als different markiert und an Personengruppen gebunden sind, wird als eine Poetik verstanden, mit der Döblin den Roman zu einer vom Exemplarischen (und nicht nur vom Einzelnen) handelnden Form umprägen wollte.
\end{abstract}

Keywords: Mehrsprachigkeit; Sprachideologie; Indexikalität; Heteroglossie; Diskursanalyse

\section{Einleitung}

Den 21. Februar 1919, den Tag, an dem Kurt Eisner in München von dem Studenten Anton von Arco ermordet wurde, beschreibt Oskar Maria Graf in seinem 1927 erschienenen Roman Wir sind Gefangene als hoch verdichtetes persönliches Erlebnis in einer gespannten und fragilen Atmosphäre:

Ich sah Zitternde, ich sah Wutblasse und Blutgierige. Überall wiederholte sich das gleiche Schreien nach Rache. Die Massen kamen ins Treiben, der Strom floß durch die Stadt. [...] Wenn jetzt einer aufgestanden wäre und hätte gerufen: ,Schlachtet die Bürger! Zündet die Stadt an! Vernichtet alles!‘ es würde geschehen sein. Die tausend kleinen Stürme hatten sich vereinigt, und ein einziger, dumpfer, dunkler, ungewisser Losbruch begann. (Graf 2008 [1927], 445-446)

Es ist ein Zustand der Liminalität (vgl. Aguirre et al. 2000), den Grafs Erzähler hier beschreibt - ja, ein Zustand der Liminalität in der Liminalität, des drohenden Umbruchs im Umbruch. Um Halt zu finden, macht Grafs Ich-Erzähler erst einmal 
das Nächstliegende - er geht nach Hause. Und dort trägt sich die folgende, für uns titelgebende Szene zu:

Als ich zu Hause ankam, traf ich meinen Zimmerherrn, der gewichtig erzählte, es seien Geiseln festgenommen worden. Er humpelte aufgeregt auf und ab und sagte in einem fort: „Jetzt müssen wir uns ranhalten!... Bloß in München ist noch was zu machen mit der revolutionären Bewegung. Draußen steht die weiße Garde, herinnen konspirieren die Konterrevolutionäre... Wenn nicht sofort alle Proleten bewaffnet werden, sind wir verratzt... Die Studenten auf der Universität haben alle ,Bravo!' gebrüllt, als Eisners Ermordung bekannt wurde... Zustand von Revolution!... Na, die Rotzbude ist schon geschlossen... Alle werden zusammengefangen... Es müssen übrigens sofort Guillotinen arbeiten.“ (Graf 2008 [1927], 449)

Der Erzähler kommentiert diesen Auftritt so:

Er redete daher wie der höchste Mann im Staat. Während er so hin und her humpelte, musterte ich ihn unvermerkt. Unwillkürlich ging mir durch den Kopf: Genau wie er ist die ganze deutsche Revolution. Sie hat auch einen Klumpfuß und hinkt. Ich mußte auf einmal laut auflachen und rief: ,Du bist ein Symbol, Mensch! Du bist ein echtes Symbol!‘ Er drehte seinen dicken Kopf rasch nach mir, hielt einen Augenblick inne und sagte kurz: ,Quatsch' nicht, Mensch! Mach’ keine Witze jetzt!‘ (Graf 2008 [1927], 449)

Doch dem Erzähler ist nicht nach Spaß zumute. Er sucht nach Orientierung und findet sie, indem er die Menschen klassifiziert. Der Zimmerherr, der sich als Revolutionär versteht und einen „revolutionären Künstlerrat“ initiiert hat, wird dabei - stellvertretend für die Künstler, die er zu vertreten beansprucht - als ungeeignet für die Revolution erwogen. Denn er hinkt nicht nur im Wortsinn, sondern auch in sozialer Hinsicht:

,Zeig’ mir deine Hände, Mann!‘ sagte ich auf einmal drohend und erfaßte seine fleischigen, kurzen Finger: ,Du hast nie eine Schaufel in der Hand gehabt, bist nie in der Fabrik gewesen!... Haben dich vielleicht die Arbeiter geholt?... Geht's ohne dich nicht?... Die Guillotinen gehören wohl für Leute wie dich!?‘ (Graf 2008 [1927], 450)

Diese Klassifikation geschieht, wie wir in diesem Beitrag zeigen wollen, nicht zuletzt aufgrund der Art und Weise, wie die Personen sprechen. Wenn der Zimmerherr „daher[redet] wie der höchste Mann im Staat“ (Graf 2008 [1927], 449), dann kann er eben nicht reden wie ein ,echter` Revolutionär. Und das wiederum kann er nach Meinung des Erzählers nicht, weil er eben kein ,echter` Revolutionär ist.

Dass der Erzähler eine solche Klassifikation, die ihm ja im Zustand der Unsicherheit Orientierung stiftet, vornehmen kann, liegt daran, dass Sprachgebräuche indexikalisch mit Personentypen und Handlungserwartungen bzw. antizipierten sozialen Praktiken verknüpft sind (vgl. zum Konzept Jaffe 2016 sowie weiter unten). 
Durch diese indexikalische Verknüpfung entsteht ein epistemischer Orientierungsrahmen, den die Soziolinguistik und Linguistische Anthropologie Sprachideologie nennt (vgl. bspw. Silverstein 1979; Kroskrity et al. 1998; Irvine und Gal 2000). Um solche Sprachideologien, die sich in metapragmatischen Praktiken (vgl. Spitzmüller 2013) manifestieren - in kommunikativen Handlungen, die kommunikative Handlungen zum Gegenstand haben - soll es in unserem Beitrag gehen.

\section{2 ,Mehrsprachigkeit‘ und Sprachideologie}

Wir möchten im Folgenden argumentieren, dass Mehrsprachigkeit vor allem dann sozial (und mithin auch literarisch) relevant ist, wenn Formen des Sprechens bzw. des Kommunizierens erstens differenziert und als verschieden wahrgenommen werden und wenn diese ,verschiedenen' Formen dann zweitens mit verschiedenen sozialen Bedeutungen verknüpft werden - ein Phänomen, dass wir im Anschluss an ein Konzept aus der Linguistischen Anthropologie, das wir in Abschnitt 3 genauer erläutern, soziale Registrierung nennen. Nur aufgrund dieser Differenzierung und Verknüpfung nämlich kann Mehrsprachigkeit zur sozialen Sinnstiftung und zur Markierung sozialer Positionen verwendet werden.

Was ,mehrsprachig‘ ist, kann vor diesem Hintergrund nicht essentiell fixiert werden. Mehrsprachig ist das, was Interpretierende als different wahrnehmen und deuten. Mit dieser Auffassung schließen wir an die neuere soziolinguistische Mehrsprachigkeitsforschung an, wie sie etwa von Busch (2013) vertreten wird. Sie hält fest:

Jeder Mensch ist mehrsprachig. Wir alle pendeln täglich zwischen verschiedenen Sprechweisen (Dialekt, geschriebene Sprache, Umgangssprache, Fachsprache...) und begegnen (in der Straßenbahn, in der Schule, in Medien, auf Reisen...) einer Vielfalt von Sprachen. (Busch 2013, Klappentext)

Das bedeutet im Umkehrschluss, dass es ,Einsprachigkeit‘ faktisch nicht gibt. Wie Busch an anderer Stelle betont:

\footnotetext{
Mit dem Satz ,Niemand ist einsprachig“ meine ich genau das: eine Erfahrung [...] des DazuGehörens oder eben nicht Dazu-Gehörens aufgrund unterschiedlicher Arten des Sprechens. Einsprachig wäre demnach nur, wer diese Erfahrung nie gemacht hat, wer sich im Sprechen nie als ,anders‘ erlebt hat. Im Vordergrund steht in dieser Sichtweise nicht die Frage, über wie viele und über welche Sprachen jemand verfügt, also ob er, um in der linguistischen Terminologie zu bleiben, neben seiner Erstsprache, der L1, auch eine L2, eine L3 oder Ln sein eigen nennen kann; noch ist das gemeint, was als innersprachliche Mehrsprachigkeit bezeichnet wird, also die Vorstellung, wonach Vartietätenbündel bestehend aus Dia-
} 
lekten, Soziolekten, Registern, Fachsprachen, Jargons usw. einer Standardsprache zugeordnet werden. Vielmehr geht es mir darum, wie sprachliche Variation dazu dienen kann, Zugehörigkeit oder Differenz zu konstruieren und vor allem, wie solche Konstruktionen als sprachliche Aus- und Einschlüsse erlebt werden. (Busch 2012, 7-8)

Dieses Mehrsprachigkeitskonzept schließt dezidiert an das Konzept der Heteroglossie an, wie es von Bachtin (1971 [1963], 202-228; 2005 [1975]) und Vološinov (1975 [1929], 177-237) geprägt wurde (vgl. Busch 2013, 10, 186-193).

,Mehrsprachigkeit" ist also nicht an ontologisch vorgegebene ,Einzelsprachen gebunden, sondern an wahrgenommene Differenz (in diesem Sinne ist, hier folgenden wir Auer 1990, auch code-switching nur als interpretatives Phänomen zu verstehen - als von Akteuren wahrgenommene und bewertete Differenz). Solche ,Einzelsprachen' bzw. das, was wir heute als solche wahrnehmen, kann man ohnehin mit guten Gründen ebenfalls als Resultat interpretativer (wenn auch langdauernder) sprachideologischer Zuschreibungen im Rahmen sprachenpolitischer Prozesse verstehen. Wie Blommaert pointiert formuliert: „In fact, the existence of ,language' and ,languages" - objects that are countable and have a name, such as English, Zulu, or Japanese - is a powerful language-ideological effect, the result of a long historical process“ (Blommaert 2006, 512). Diese Zuschreibungen sind, wie etwa Gal (2006) zeigt, eng an die europäische Nationenbildung gekoppelt, weshalb man mit den Worten dieser Sprachanthropologin zugespitzt sagen kann:

It may seem odd to say so, but ,language، was invented in Europe. Speaking is a universal feature of our species, but ,language' as first used in Europe and now throughout the world is not equivalent to the capacity to speak, but presumes a very particular set of features. Languages in this limited sense are assumed to be nameable (English, Hungarian, Greek), countable property (one can ,have' several), bounded and differing from each other, but roughly inter-translatable, each with its charming idiosyncrasies that are typical of the group that speaks it. The roots of this language ideology go back to the European Enlightenment and the Romantic reaction that followed. (Gal 2006, 14)

\section{Indexikalität und Registrierung}

Wahrgenommene Differenz nun aber ist soziolinguistisch dann interessant, wenn sie mit Verhaltenserwartungen und Interpretationsmodellen verkoppelt ist. Diese Verkopplungen sind indexikalisch in dem Sinne, dass sie Kommunikationsformen an Kontexte binden (vgl. Blommaert 2005, 11-12). Indexikalische Verkopplungen entstehen durch reflexive Assoziation von Sprachgebräuchen an vergangene kommunikative Praktiken, in denen wir die Sprachgebräuche erfahren haben. Wie Maas es treffend formuliert: 
[...] jeder sprachliche Ausdruck in unserer Sprachbiographie [ist] durch den Kontext indiziert [...], in dem wir ihn kennengelernt haben - jenseits seiner in Wörterbüchern explizierten Bedeutung bedeutet/bezeichnet jeder Ausdruck eben immer auch reflexiv die Situation, deren Artikulation er ermöglicht (hat). (Maas 1989, 168)

Daher können, wie Maas an anderer Stelle ausführt, Formen des Handelns umgekehrt soziale Praktiken indizieren; „,...] die soziale Praxis, und damit eben auch jede Sprachpraxis, [ist] reflexiv [...]: die Form jeder Praxis, also die Form, in der die jeweilige Praxis artikuliert worden ist, kann selbst zum Zeichen für diese Praxis werden“ (Maas 1985, 73). Das heißt: Weil wir kommunikative Formen in bestimmten Kontexten erleben (vgl. zum Konzept des Spracherlebens Busch 2017), verbinden wir die Formen mit den Kontexten. Diese indexikalische Verknüpfung von Formen, Kontexten, Akteuren und Praktiken ist es, worauf das Konzept der sozialen Registrierung abzielt, das wir nun etwas genauer erläutern möchten (vgl. dazu ausführlicher Spitzmüller 2013).

Register sind nach einer einschlägigen Definition von Agha $(2007,135)$,"culture-internal models of personhood linked to speech forms". Sie bestehen aus, wie Agha (2007, 235) es nennt, sozialen Emblemen (social emblems); das sind indexikalische Zeichen, die typisierte Personen (personae) und Praktiken - also antizipierte soziale Konzepte - als Referenzobjekt haben. Den Prozess, in dem solche Verknüpfungen etabliert und sozial distribuiert werden, nennt Agha (2007, 81) soziale Registrierung (social enregisterment). Register-Verkopplungen umfassen nicht nur Sprachgebrauch im engen Sinn, sondern schließen auch dezidiert Kommunikation als körperliches Verhalten (wie das ,Hin- und Herhumpeln“ von Grafs Zimmerherrn) ein (vgl. Agha 2007, 235) - Bucholtz und Hall $(2016,178)$ sprechen in diesem Zusammenhang von „embodied indexicality“.

Solche indexikalischen Kopplungen finden sich in den Quellen, die wir durchgesehen haben, vielfach. Vor allem zwei Personentypen werden einander gegenübergestellt: Auf der einen Seite die ,Intellektuellen', darunter auch ,Literaten' und (wie es mehrfach explizit und zumeist abwertend heißt),Professoren“ bzw. ,Bourgeois', auf der anderen Seite die ,echten Arbeiter', ,einfachen Leute‘ bzw. ,Proletarier' (vgl. bspw. Dorst 1966, 7-8, 32, 36, 65, 66, 70, 73, 166). Die beiden Personentypen zeichnen sich jeweils durch differenzierbaren, sprachideologisch evaluierten Stil aus. Besonders deutlich zeigt sich dies in den Positionierungspraktiken der Sozialdemokratie gegenüber dem Marxismus und den gegengerichteten Abgrenzungen der Marxisten gegenüber der Sozialdemokratie. Die Marxisten (und auch Marx selbst) werden hierbei wiederholt von sozialdemokratischen Akteuren als ,phantasielos' und ,unpoetisch' charakterisiert. Exemplarisch hierfür ist die folgende Evaluation Landauers: 
Niemals wird der Sozialismus aus dem Kapitalismus ,erblühen', wie der Undichter Marx so lyrisch gesungen hat. [...] [W]enn es je phantasielose Phantasten gegeben hat, so sind es die Marxisten [...]. [...] Die Proletarier haben nichts zu verlieren; sie haben eine Welt zu gewinnen! Wie schön, wie wirklich dichterisch ist dieses Wort (das weder von Marx noch von Engels stammt) und wie viel Wahres sollte darin sein. (Gustav Landauer, Aufruf zum Sozialismus 1919; aus Dorst 1966, 16-18)

Der Personentypus des ,wohlsprechenden Literaten' dient hier als Positionierungsanker, zu dem sich die Sozialdemokraten hin ausrichten, während sie die Marxisten weit entfernt von diesem Pol positionieren - und mithin sich selbst weit entfernt von den Marxisten (vgl. für eine linguistische Modellierung solcher tripolarer Positionierungen Du Bois 2007). Konsequenterweise sehen sich die sozialdemokratischen Führer, wie im Folgenden Eisner, von ihren Opponenten gerade deswegen (zu Unrecht) angegriffen, weil sie, gutes Deutsch`schreiben und ,Dichter' sind:

Seit der Zeit habe ich die Presse gegen mich [...]. Deshalb wurde die Losung ausgegeben: Feste druff auf diesen Ideologen, auf diesen Phantasten, auf diesen Dichter. Ich teile dieses Unglück mit dem französischen Minister Clemenceau, der auch ein Dichter ist. Nur bei uns herrscht noch die Meinung, daß jemand, der gutes Deutsch schreibt, wenn nicht ein Narr, so mindestens ein schlechter Politiker ist. (Kurt Eisner, Wahlrede 1919; aus Dorst 1966, 36)

Und tatsächlich verspotten auf der anderen Seite marxistische Akteure die ,bildungsbürgerlichen' und ,schöngeistigen' Praktiken der Sozialdemokraten. So wird in der ersten Ausgabe der vom Münchner Ortsverein des Spartakusbundes herausgegebenen „Flugzeitung“ Münchner Rote Fahne mit direktem Blick auf Eisner argumentiert:

Die kommunistische Partei kennt keine Politik der ,Kunst‘, keine Politik der ,Phantasie“, keine Politik der ,Kabinette', keinen Kuhhandel mit seinem Todfeind, der Bourgeoisie. [...] Die ,Neue Zeitung‘, die ein Luxusunternehmen Eisners ist, [...] gehört auf die Oktoberwiese. Diese journalistische Mumie ist nur durch eines eine immerhin nicht gewöhnliche Erscheinung: durch die berückende Zartheit ihrer literarischen Blüten und die famose Fähigkeit, aus der Politik ein Kaffeekränzchen zu machen. [...] Die unartige, proletarische Kritik aber ist diese: es ist ein Skandal, daß eine Partei, in einer Zeit, in der sie eine schwere Krisis durchmacht, in vollster Auflösung begriffen ist, deren Führer wie der Krebs, der Hecht und Schwan in Lafontaines Fabel den festgefahrenen Karren der Revolution aus dem Dreck ziehen wollen, als Sprachrohr ihrer Anschauung einen politischen Eunuchen in die Welt setzt! (Münchner Rote Fahne 1 [15. Januar 1919]: 1; aus Scheringer et al. 1978) 
Und in einem Flugblatt der KPD-Ortsgruppe München heißt es:

Die Bourgeoisie ist kein Kaffeekränzchen [...]. [...] [N]icht Dank ernten sie bei den Kapitalisten für ihre Bücklinge und Kratzfüße, sondern nur offenen Spott, Hohn und Verachtung, wie es ihnen auch gebührt. (Flugblatt der KPD Ortsgruppe München, undatiert; aus Scheringer et al. 1978)

Die Beispiele verdeutlichen, wie umfassend registriert die antizipierten Praktiken der hier distanzierend konstruierten Personentypen offenbar sind - die Art, wie sie (angeblich) sprechen (,die berückende Zartheit ihrer literarischen Blüten“), die mit ihnen assoziierten sozialen Gattungen („Kaffeekränzchen“) und auch ihr körperliches Verhalten („Bücklinge und Kratzfüße“). Die Aushandlung sozialer Positionen, die dieser (und jeder) Revolution ja wesentlich ist, ist also offensichtlich eng verbunden mit metapragmatischen Verkopplungen von kommunikativem Verhalten bzw. Handlungspotenzen und Personentypen, also mit als different wahrgenommenen, ideologisch salient gemachten akteursgebundenen kommunikativen Praktiken (Registern). Der zweite Teil unseres Beitrags geht dieser Spur am Beispiel eines ausgewählten Textes zur Revolution 1918/19 weiter nach.

\section{Alfred Döblins November 1918 - eine Quelle zweiter Ordnung}

Die Quelle, anhand der die bis zu diesem Punkt entfalteten Überlegungen nun weiter vertieft werden sollen, ist eine literarische: Alfred Döblins Romantetralogie November 1918, geschrieben zwischen 1937 und 1943, in den Jahren von 1939 bis 1950 in Teilen publiziert (vgl. Sander 2008, 413), zusammenhängend jedoch erst 1978 (vgl. Blume 1991, 169) sowie 1991 als Teil der Döblin-Werkausgabe (vgl. Schoeller 2011, 579). Für die Wahl dieser Quelle spricht nicht nur ihr Gegenstand, die Deutsche Revolution, sondern besonders, dass sie von jener Form der Mehrsprachigkeit geprägt ist, von der in den Abschnitten 1-3 vorwiegend in theoretischen Registern die Rede war. Das Evozieren verschiedener Formen des Sprechens, die als different markiert bzw. wahrgenommen und an Personengruppen gebunden werden, zählt zu den stilistischen Besonderheiten dieses Monumentalwerks und tritt uns in ihm als ein literarisches Verfahren entgegen. In der nun anschließenden Analyse gilt es, die Erzeugung dieser unterschiedlichen Formen des Sprechens bei Döblin unter Rückgriff auf die hier vorgestellten Konzepte aufzuweisen (Abschnitt 5) sowie diese Heteroglossie als ein stilistisches Verfahren zu kontextualisieren (Abschnitt 6). Das bedeutet zum einen ein Modell der Soziolinguistik anhand eines literarischen Textes zu erproben, zum anderen die Frage 
nach der Funktion zu stellen, welche die Ko-Präsenz verschiedener Formen des Sprechens in diesem literarischen Text erfüllt. Diese Untersuchungsperspektive ist als eine kritische Reaktion darauf zu verstehen, literarische Mehrsprachigkeit lediglich aufzuweisen ohne aber danach zu fragen, welche Funktion ihr in einer konkreten literaturgeschichtlichen Situation zukommt.

Dieser zweiteiligen Analyse sei vorausgeschickt, dass Döblins Roman im Unterschied zu den zuvor diskutierten Quellen Jahrzehnte nach den revolutionären Ereignissen von 1918 verfasst wurde. Der von Walter Muschg begründeten Ausgabe der Werke Döblins ist eine präzise Rekonstruktion jener Quellen zu entnehmen, auf die der 1878 in Stettin geborene Autor bei der Ausarbeitung seiner Tetralogie zurückgriffen hat (vgl. Döblin 1991 [1937-1943], Bd. 3, 783-787). Es ist weiter bekannt, dass der während des Abschluss seines Romans bereits im kalifornischen Exil lebende Döblin mit Problemen konfrontiert war, an Zeitungen und Zeitschriften aus der Revolutionszeit zu gelangen. Seinen Briefen dieser Jahre ist zu entnehmen, wie er seinen in New York lebenden Freund Hermann Kesten sowie eine Bibliothekarin der New York Public Library darum ersucht, ihm Dokumente aus der Revolutionszeit nach Los Angeles zu schicken (vgl. Schoeller 2011, 578). Relevant sind diese Hinweise auf die schwierige Textgenese von November 1918 zumindest aus zwei Gründen: Als ein Werk der deutschsprachigen Exilliteratur, 1937 in Paris begonnen, 1943 in den USA abgeschlossen, haben wir es hier erstens mit Mehrsprachigkeit auch auf Ebene der Textproduktion und -distribution zu tun, die unter den prekären Umständen des amerikanischen Exils erfolgte (vgl. Domokos 2011). Zweitens stellt November 1918 im Rahmen unserer Fallstudie dieser Punkt ist für die nun zu entfaltende Perspektive besonders relevant - eine Quelle zweiter Ordnung dar. Anders gesagt: Quellen aus jener Zeit, der unsere Analyse bis zu diesem Punkte galt, sind von Döblin aufgegriffen und weiterverarbeitet worden.

\section{5 ,Mehrsprachigkeit' als Mittel der sozialen Stratifikation}

Es ist weitgehend bekannt, dass November 1918 ein gesellschaftlich breites Panorama von Einzelschicksalen entwirft (vgl. Blume 1991, 77), in dem sich fiktive Charaktere, bspw. der Kriegsheimkehrer Friedrich Becker, der Dramatiker Erwin Stauffer, gleichermaßen tummeln wie historische Figuren, bspw. Karl Liebknecht, Rosa Luxemburg oder Woodrow Wilson. Für die Struktur des Textes bezeichnend ist weiter ein häufiger Wechsel zwischen verschiedenen sozialen Kontexten, Bürgertum und Proletariat, sowie Personengruppen, Soldaten, Politikern und 
Matrosen - wodurch ein Text entsteht, dessen Gestaltung der Revolution auf kein einzelnes Milieu beschränkt bleibt.

Wie aber gelingt es diesem Text, eine derart breite soziale Stratifikation zu erzeugen, und wie werden die einzelnen Figuren mit diesen sozialen Milieus verbunden? Eine entscheidende, wenn auch nicht die einzige, Rolle spielt in diesem Zusammenhang die Figurenrede. Möchte man unter Zuhilfenahme der zuvor vorgestellten soziolinguistischen Konzepte präzisieren, wie dieser Text aus Figurenrede unterschiedliche soziale Kontexte bildet, wird es möglich, zumindest zwei Darstellungstechniken zu unterscheiden. Wir beschränken uns dabei auf Stichproben, die für die Poetik des Textes insgesamt stehen. ${ }^{1}$

Eine erste (und in zahlreichen anderen Romanen verfolgte) Technik besteht darin, die Figuren nicht nur bestimmten Berufs- und damit Gesellschaftsgruppen zuzuordnen, sondern diese Zuordnung durch ihre Form des Sprechens auch auszugestalten. Einer der zentralen Charaktere, Friedrich Becker, der, was mit Blick auf die bereits aufgewiesene Intellektuellenkritik um 1917/18 besonders interessant ist, vom Erzähler als „Altphilologe“ (Döblin 2008 [1937-1943], Bd. 1, 121) markiert wird, ist im ersten Teil in einem Kriegslazarett liegend dargestellt. Seine Rede ist in dieser Szene signifikant von jenen der anderen, etwa der eines Leutnants oder einer Krankenschwester, unterschieden. Es ist eine Figurenrede, in der Vergleiche zwischen Gegenwart und antiker Mythologie gebildet werden (vgl. Döblin 2008 [1937-1943], Bd. 1, 121) oder in der, wie in folgender Passage, ein Klassiker-Zitat (aus Schillers Wilhelm Tell) aufgerufen wird:

Sie [d. i. die Krankenschwester] betrachtete ihn aufmerksam. ,Sie blicken auf meine Kurve, Schwester Hilde. 36.8 - das ist nichts. Ich mache Gebrauch von dem Recht, von dem Schiller gesprochen hat: Nein, eine Grenze hat Tyrannenmacht. Wenn der Gedrückte nirgends recht kann finden, wenn unerträglich wird die Last, greift er hinauf getrosten Muts in den Himmel und holt herunter seine ewigen Rechte, die droben hangen, unveräußerlich und unzerbrechlich, wie die Sterne selbst. Der alte Urstand der Natur kehrt wieder.' (Döblin 2008 [1937-1943], Bd. 1, 125-126)

Anschließend an Agha lässt sich behaupten, dass sich diese Technik auf eine bereits stark registrierte indexikalisch-soziale Bedeutung bezieht (vgl. Spitzmül-

1 Der Vollständigkeit halber sei erwähnt, dass wir in November 1918 noch eine Reihe anderer Techniken finden, die nicht an Figurenrede gebunden sind, und dennoch soziale Kontexte evozieren bzw. indexikalische Verknüpfungen zwischen diesen und den Figuren erzeugen. Diese könnte man im weitesten Sinne auf der Ebene der Narration ansiedeln: in Techniken der Fokalisierung bzw. Perspektivierung oder des szenischen Erzählens (bspw. Döblin 2008 [1937-1943], Bd. 1, 296-297). 
ler 2013, 266); in dem Sinne, dass eine spezifische Form des Sprechens (hier: das Sprechen unter Bezugnahme auf einen Klassiker der deutschsprachigen Literatur) sozial bereits so verortet ist, dass sie dazu verwendet werden kann, um die vom Erzähler gemachte Zuordnung einer Figur zu einer gesellschaftlichen Gruppe (hier: den Gelehrten) zu ,stilisieren'. In theoretischen Registern Bachtins gesprochen, jenes Theoretikers, auf den der Begriff der ,Stilisierung' rekurriert, bedient sich Döblins Erzähler hier des „fremden Worts in der Richtung seiner eigenen Absichten“ (Bachtin 1971 [1929], 215). Genauer gesagt bedient er sich dessen, um die soziale Position seiner Figur zu markieren.

Einer weiteren Technik, um aus Figurenrede unterschiedliche, miteinander rivalisierende soziale Kontexte zu evozieren, begegnen wir dort, wo Romanfiguren die Rede anderer Romanfiguren aufgreifen, kommentieren, bewerten und einer Gruppe zuordnen. Wir befinden uns in der folgenden Passage im ersten Teil des Romans: Zwei aus dem Weltkrieg heimgekehrte Soldaten, Hans Bruch und Johannes Maus, sowie zwei weitere Figuren, die keinen Namen tragen, betreten ein Berliner Lokal, in dem ,in einem Hinterzimmer eine Handvoll angeblicher Spartakusleute [...] gegenseitig politische Ansprachen [hielten]“. (Döblin 2008, Bd. 1, 295) Die vier Männer sind offensichtlich in der Hoffnung auf ein warmes Essen in das Lokal getreten, werden nun aber Zeugen einer politischen Debatte. Es dauert nicht lange bis die vier beschließen, das Lokal wieder zu verlassen:

Da zogen die beiden den Älteren heraus und ließen sich an der Weberstraße ungeheuer über die Quatschköpfe drinnen aus. Wozu Hans sagte: ,Wenn wir draußen mit einem Angriff so lange gewartet hätten wie die und alles verraten hätten, hätten sie uns schon nach einem Monat gehabt, und keiner von uns könnte heute ,pieps‘ sagen. 'Und da laufen noch', wütete der zweite, ,solche Esel und Gelehrte mit Brillen. ' Der dritte: ,Die Gelehrten sind überall die dämlichsten. Wie man sich mit denen einlassen kann, habe ich zeit meines Lebens nicht verstanden. ' Hans ungeduldig vor der Kirche: ,Also was soll sein?‘ Worauf der zweite ihm das Wort, das sie drin gehört hatten, ,expropriieren', ins Ohr flüstere. Hans ärgerlich: ,Mach keinen Blödsinn. Wo willst Du Abend essen? Willst du dich wieder bei der Suppenküche anstellen und Backebackekuchen machen?` Aber der zweite wußte, was er sagte. Er hatte gerade in dem Lokal Fühlung mit zwei anderen bekommen, zwei Westpreußen, deren Heimatstädte zerstört waren und die dabei waren, sich in Berlin schadlos zu halten. Die hatten frisch ,Expropriierungsgruppen‘ gebildet. (Döblin 2008 [1937-1943], Bd. 1, 296)

Auffällig ist, wie die im Lokal Debattierenden erst in der Stimme des Erzählers als „Quatschköpfe“ und dann in Figurenrede als „Esel und Gelehrte mit Brillen“ bezeichnet und damit abgewertet werden. Daran anschließend greift eine der Figuren das von den Gelehrten im Lokal vernommene Wort „expropriieren“ auf, dessen Gebrauch von Hans mit dem Satz kommentiert wird: „Mach keinen Blödsinn“. Die Entwertung der Gelehrten wird durch die Kommentierung ihrer Sprechweise als unpassend weiter verstärkt. Wir beobachten hier eine metaprag- 
matische Distanzierung von den Gelehrten, die zugleich eine Solidarisierung der Soldaten bedeutet. Entscheidend ist - und in diesem Punkt vermögen die unsere Argumentation leitenden linguistischen Konzepte Nuancen dieses literarischen Textes zu erhellen -, dass mit den Koppelungen bestimmter Redeweisen an Personengruppen (hier: Fremdwörter an Gelehrte) auch Bewertungen bezüglich ihrer Rolle in der Revolution einhergehen: „Wenn wir draußen mit einem Angriff so lange gewartet hätten wie die und alles verraten hätten, hätten sie uns schon nach einem Monat gehabt, und keiner von uns könnte heute ,pieps‘ sagen.“ Es wird hier eine Opposition erzeugt zwischen jenen, die nur debattieren und Fremdwörter verwenden, und jenen, die handelnd aktiv werden können. Sie erinnert an den Gegensatz zwischen Agitation/Aktion bzw. Kaffeekränzchen/Revolution, wie er schon in einem früheren Beispiel (vgl. das Zitat aus der Münchner Roten Fahne) deutlich wurde. In Döblins Roman wird dieser Gegensatz durch die räumliche Strukturierung der Szene, drinnen/draußen, mit narrativen Mitteln weiter ausgestaltet und dadurch verstärkt.

So kurz die zuletzt diskutierte Szene also auch sein mag, lässt sie sehr präzise anhand von kommunikativen Praktiken eine jener Konfliktlinien hervortreten, welche die Debatten nicht nur über das Scheitern der Deutschen Revolution, sondern der 1930er Jahre überhaupt bestimmt haben: jene zwischen Denken und Handeln. An diesen Begriffen konkretisieren sich gewisse pejorative Vorstellungen vom Gelehrten bzw. Intellektuellen, auf die epochemachende Texte der 1930er Jahre antworten: Robert Musils Roman Der Mann ohne Eigenschaften (1930/32), in dessen Mittelpunkt eine Intellektuellenfigur steht, Karl Mannheims Ideologie und Utopie (1929), in dessen letztem Abschnitt, „Die gegenwärtige Konstellation“, das Theorem der ,freischwebenden Intelligenz entfaltet wird, sowie Döblins Manifest Wissen und Verändern! ([1931] 1972), in dem die Frage verhandelt wird, welche Bedeutung intellektueller Arbeit in der gegenwärtigen Gesellschaft zukomme. Eine Analyse von metapragmatischen Äußerungen in der Literatur erweist sich so als ein vielversprechendes und noch $\mathrm{zu}$ wenig aufgegriffenes Instrument, um Text-Kontext-Beziehungen zu untersuchen, d.h. literarische Texte präziser in ihren diskursiven Kontexten zu situieren.

\section{6 ,Mehrsprachigkeit‘ und Döblins Romanpoetik der 1920er/1930er Jahre}

Von der Prämisse ausgehend, dass mit dem Aufweis von Mehrsprachigkeit, auch wenn sie anders konzeptualisiert ist als in dem vorliegenden Beitrag, noch nicht viel gewonnen ist, gilt es abschließend zu fragen, welche Funktionen der durch 
verschiedene Techniken erzeugten Mehrsprachigkeit nun also zukommt. Eine Methode, um zumindest eine dieser Funktionen näher zu fassen, ist es, Döblins Verfahren, Formen des Sprechens als different zu markieren und an soziale Gruppen zu binden, vor der Folie seiner poetologischen Äußerungen dieser Jahre zu lesen. In den späten 1920er und 1930er Jahren hat Döblin auch in seinen programmatischen Texten versucht, eine Form des Romans zu profilieren, in der jeweils „das Exemplarische des Vorgangs und der Figuren“ (Döblin 1989a [1929], 218) dargestellt ist - so eine Formulierung aus dem Aufsatz Der Bau des epischen Werks von 1929. Nur so, argumentiert Döblin, könne wieder jene ,epische Wahrheit' entstehen, die er in einer Praxis des Romans seiner Zeit vermisst. Döblins Anspruch, jeweils das Exemplarische sichtbar zu machen, ist in der Forschung als „kollektivierende Darstellung“ (Blume 1991, 84) bezeichnet worden. Der Einzelmensch steht - etwas robust formuliert - in Döblins epischem Werk dieser Jahre sehr oft für eine Personengruppe. Diese Poetik, die etwas später auch in der Der historische Roman und wir von 1936 (Döblin 1989b [1936], 297) anklingt, lässt sich mit Blick auf November 1918 nicht zuletzt an den Titeln der Einzelbände ablesen, die, wohlgemerkt nicht nur, aber vorwiegend, Personengruppen aufrufen: Bürger und Soldaten, Verratenes Volk, Heimkehr der Frontruppen. Wenn also Döblin in November 1918 anhand verschiedener Figuren verschiedene Sprachen aufruft und diese an unterschiedliche Milieus bindet, könnte diese Technik auch dazu dienen, nicht nur vom Einzelnen, sondern immer auch vom Einzelnen als Pars pro Toto einer ganzen Gruppe, vom Besonderen bzw. Exemplarischen als Allgemeines zu handeln. In eine knappe These gefasst, lässt sich Folgendes behaupten: Die literarische Mehrsprachigkeit dieses Textes, der die Indexikalität sprachlicher Formen nutzt, um Figuren als Signifikanten sozialer Positionen zu zeigen, dient Döblin dazu, den zeitgenössischen Roman zu einer vom ,Exemplarischen“ handelnden Gattung umzuprägen. Sie ist ihm ein Mittel, um dem Roman durch Anschluss an die Tradition des Epos neue Geltung zu verschaffen. ${ }^{2}$

\section{Fazit und Ausblick}

Mit Blick auf die in unserer ICLA-Sektion im Zentrum stehenden Fragen nach der Rolle von Mehrsprachigkeit in der Literatur lassen sich unsere Einsichten nun folgendermaßen bündeln: 1) Mehrsprachigkeit möchten wir im Anschluss an die vorgestellten Konzepte der Soziolinguistik als das Ergebnis einer Wahr-

2 In dieselbe Richtung weist schließlich auch ein anderes Verfahren des Textes, nämlich, das historische Geschehen der Deutschen Revolution im Lichte des (Antigone-)Mythos darzustellen. 
nehmung und Deutung der Akteure verstehen, die es von Seiten der Wissenschaft zu rekonstruieren gilt. Dabei ist 2) im Falle der literarischen Mehrsprachigkeit, verstanden als Resultat einer fiktionsinternen Wahrnehmung und Deutung von Redeweisen seitens der Figuren und des Erzählers, immer auch nach ihrer Funktion zu fragen, die im Falle Döblins in einer Umprägung der Gattung des zeitgenössischen Romans liegt, der nicht nur vom Einzelfall, sondern vom Exemplarischen handeln soll. Schließlich lässt sich 3) aus dem Dargestellten noch ein Wort zur besonderen Relevanz von Revolutionsdiskursen im Zusammenhang mit der Erforschung von Mehrsprachigkeit sagen. Als Phasen der Liminalität, wie wir es eingangs formuliert haben, als Zeiten der Ungewissheit, in denen soziale Grenzen verletzlich werden und soziale Ordnungen verrutschen, stellen Phasen der Revolution für das hier vertretene Konzept ,sozialer Mehrsprachigkeit‘ ein besonders ergiebiges Forschungsgebiet dar. Was diese Verunsicherung sozialer Grenzen für die konkrete Textanalyse bedeutet - hierzu möchten wir abschließend zwei Vermutungen äußern, die einen Ausblick auf unsere nächsten Arbeitsschritte geben. Eine erste Vermutung ist, dass die sozialen Klassen wegen dieser Verunsicherung in den kommunikativen Handlungen besonders vehement affirmiert werden, eine zweite, dass die kommunikativen Handlungen selbst unsicherer werden; in dem Sinne, dass bspw. die soziale Indexikalität von Zeichen verrutscht und dadurch Markierungen schwieriger werden. Unsere Überlegungen gehen aktuell dahin, dass beides der Fall ist und dass diese Prozesse eng aufeinander bezogen sind. In diese Richtung jedenfalls weisen die nächsten Schritte unserer Erforschung von Revolutionsdiskursen, in die sich die hier vorgelegte Skizze zur sozialen Mehrsprachigkeit in literarischen und nicht-literarischen Quellen einordnet.

\section{Literaturverzeichnis}

Agha, Asif. Language and Social Relations. Cambridge: Cambridge University Press, 2007.

Aguirre, Manuel, Roberta Quance, und Philip Sutton. Margins and Thresholds. An Enquiry into the Concept of Liminality in Text Studies. Madrid: The Gateway Press, 2000.

Auer, Peter. „A Discussion Paper on Code-Alternation“. Papers for the Workshop on Context, Methodology and Data, Basel, Jan 12-13, 1990. Hg. ESF Network on Code-Switching and Language Contact. Straßburg: European Science Foundation, 1990. 69-91.

Bachtin, Michail M. Probleme der Poetik Dostoevskijs. Übers. v. Adelheid Schramm. München: Hanser, 1971 [zuerst russ.: Problemy poetiki Dostoevskogo. Moskau: Sovetskij pisatel', 1963; 2. erw. Aufl. des Buchs Problemy tvorchestva Dostoevskogo, Leningrad: Priboj, 1929].

Bachtin, Michail M.: „Das Wort im Roman“. Michail M. Bachtin: Die Ästhetik des Wortes. Hg. u. mit einer Einl. vers.v. Rainer Grübel. Übers.v. Rainer Grübel und Sabine Reese. 7. Aufl. Frankfurt/M.: Suhrkamp, 2005. 154-300 [zuerst russ.: Slovo v romane. In: Michail M. 
Bachtin. Voprosy literatury i ėstetiki. Issledovanija raznych let. Moskau: Chudožestvennaja literature, 1975. 72-233; geschrieben 1934/35]

Behschnitt, Wolfgang, Sarah De Mul und Liesbeth Minnaard (Hgg.). Literature, Language, and Multiculturalism in Scandinavia and the Low Countries, Amsterdam u. a.: Brill, 2013.

Blommaert, Jan. Discourse. A Critical Introduction. Cambridge: Cambridge University Press, 2005.

Blommaert, Jan. „Language Ideology“. Encyclopedia of Language \& Linguistics. Hg. Keith Brown. 2. Aufl. Oxford: Elsevier, 2006. Bd. 6. 510-522.

Blume, Jürgen. Die Lektüren des Alfred Döblin. Zur Funktion des Zitats im Novemberroman. Frankfurt/M. u. a.: Peter Lang, 1991.

Bucholtz, Mary, und Kira Hall: „Embodied Sociolinguistics“. Sociolinguistics. Theoretical Debates. Hg. Nikolas Coupland. Cambridge: Cambridge University Press, 2016. 173-199.

Busch, Brigitta. „Expanding the Notion of the Linguistic Repertoire: On the Concept of Spracherleben - The Lived Experience of Language“. Applied Linguistics 38.3 (2017): 340-358.

Busch, Brigitta. Das sprachliche Repertoire oder Niemand ist einsprachig. Vorlesung zur Verleihung der Berta-Karlik-Professur an der Universität Wien. Klagenfurt/Celovec: Drava, 2012.

Busch, Brigitta. Mehrsprachigkeit. Wien: Facultas, 2013.

Döblin, Alfred. „Wissen und Verändern! Offene Briefe an einen jungen Menschen“. Der deutsche Maskenball von Linke Poot. Wissen und Verändern! Hg. Adolf Muschg. Olten und Freiburg i. Br.: Walter, 1972. 125-266 (Alfred Döblin. Ausgewählte Werke in Einzelbänden. 28 Bde. Bd. 21) [zuerst: Berlin: S. Fischer, 1931].

Döblin, Alfred. „Der Bau des epischen Werks“. Schriften zu Ästhetik, Poetik und Literatur. Hg. Erich Kleinschmidt. Olten und Freiburg i. Br.: Walter, 1989a. 215-245 (Alfred Döblin. Ausgewählte Werke in Einzelbänden. 28 Bde. Bd. 16) [zuerst in: Die Neue Rundschau 1.40 (1929): 527-551].

Döblin, Alfred. „Der historische Roman und wir“. Schriften zu Ästhetik, Poetik und Literatur. Hg. Erich Kleinschmidt. Olten und Freiburg i. Br.: Walter, 1989b. 291-316 (Alfred Döblin. Ausgewählte Werke in Einzelbänden. 28 Bde. Bd. 16) [zuerst in: Das Wort 1.4 (1936): 56-71].

Döblin, Alfred. November 1918. Eine deutsche Revolution. Erzählwerk in drei Teilen. Hg. Werner Stauffacher. 4 Teilbde. Olten: Walter, 1991 (Alfred Döblin. Ausgewählte Werke in Einzelbänden; Bd. 29) [verfasst 1937-1943].

Döblin, Alfred. November 1918. Eine deutsche Revolution. Erzählwerk in drei Teilen. 4 Bde. Frankfurt/M.: S. Fischer, 2008 [verfasst 1937-1943].

Domokos, Johanna. „Differentiation of Cultural Interferences of the (Uralic) Literary Field“. Multilingualism and Multiculturalism in Finno-Ugric Literatures. Hg. Johanna Laakso und Johanna Domokos. Münster: LIT, 2011. 12-25.

Dorst, Tankred (Hg.). Die Münchner Räterepublik. Zeugnisse und Kommentar. Mit einem Komm. von Helmut Neubauer. Frankfurt/M.: Suhrkamp, 1966.

Du Bois, John W. „The Stance Triangle“. Stancetaking in Discourse. Subjectivity, Evaluation, Interaction. Hg. Robert Englebretson. Amsterdam und Philadelphia: Benjamins, 2007. 139-182.

Gal, Susan. „Migration, Minorities and Multilingualism: Language Ideologies in Europe“. Language Ideologies, Policies and Practices. Language and the Future of Europe. Hg. Clare Mar-Molinero and Patrick Stevenson. Houndsmill: Palgrave Macmillan, 2006. 13-27. 
Graf, Oskar Maria. Wir sind Gefangene. Ein Bekenntnis. 11. Aufl. München: dtv, 2008 [zuerst: Wir sind Gefangene: ein Bekenntnis aus diesem Jahrzehnt. München: Drei Masken, 1927].

Irvine, Judith T., und Susan Gal. „Language Ideology and Linguistic Differentiation“. Regimes of Language: Ideologies, Polities, and Identities. Hg. Paul V. Kroskrity. Oxford: Currey, 2000. 35-84.

Jaffe, Alexandra. „Indexicality, Stance and Fields in Sociolinguistics“. Sociolinguistics. Theoretical Debates. Hg. Nikolas Coupland. Cambridge: Cambridge University Press, 2016. 86-112.

Kiesel, Helmuth. Literarische Trauerarbeit. Das Exil- und Spätwerk Alfred Döblins. Tübingen: Niemeyer, 1986.

Kuhlmann, Anne. Revolution als ,Geschichte‘: Alfred Döblins „November 1918“. Eine programmatische Lektüre des historischen Romans. Tübingen: Niemeyer, 1997.

Kroskrity, Paul V., Bambi B. Schieffelin und Kathryn A. Woolard (Hgg.). Language Ideologies: Practice and Theory. New York: Oxford University Press, 1998.

Laakso, Johanna. „Linguistic Approaches to Finno-Ugric Literary Multilingualism“. Multilingualism and Multiculturalism in Finno-Ugric Literatures. Hg. Johanna Laakso und Johanna Domokos. Münster: LIT, 2011. 26-36.

Laakso, Johanna. Multilingualism in Minority Literatures from a Finno-Ugric Linguist's Point of View. Paper presented at the intersection of the conferences Multilingualism in Baltic Sea Europe and Research Agendas in Literary Linguistics at the Johannes Gutenberg University of Mainz, April 15, 2015. https://www.academia.edu/11967564/Multilingualism_in_ minority_literatures_from_a_Finno-Ugric_linguist_s_point_of_view (14. Juli 2016).

Maas, Utz. „Konnotation“. Politische Sprachwissenschaft. Zur Analyse von Sprache als kultureller Praxis. Hg. Franz Januschek. Opladen: Westdeutscher Verlag, 1985. 71-95.

Maas, Utz. „Sprache im Nationalsozialismus. Analyse einer Rede eines Studentenfunktionärs“. Sprache im Faschismus. Hg. Konrad Ehlich. Frankfurt/M.: Suhrkamp, 1989. 162-197.

Müller-Wille, Klaus. „,Svenska för inhemska‘. Zu einer Poetik der Fremdsprache bei Ilmar Laaban und Alexander Weiss“. Literatur der Migration - Migration der Literatur. Hg. Karin Hoff. Frankfurt/M. u. a.: Peter Lang, 2008. 49-70.

Sander, Gabriele. „Alfred Döblin, November 1918. Eine deutsche Revolution“. Döblin, Alfred. November 1918. Eine deutsche Revolution. Erzählwerk in drei Teilen. Bd. 1. Frankfurt/M.: Fischer, 2008. 413-415 [verfasst 1937-1943].

Scheringer, Richard, Conrad Schuhler und Michael Führer (Hgg.). 60 Jahre Münchner Räterepublik. Illustrierte Geschichte, Augenzeugenberichte, Dokumente. München: DKP Vorstand Südbayern, 1978 [Beilage ohne Paginierung].

Schoeller, Wilfried F. Alfred Döblin. Eine Biographie. München: Hanser, 2011.

Silverstein, Michael. „Language Structure and Linguistic Ideology“. The Elements: A Parasession on Linguistic Units and Levels. Hg. Paul R. Cline, William Hanks, Carol Hofbauer. Chicago: Chicago Linguistic Society, 1979. 193-247.

Spitzmüller, Jürgen. „Metapragmatik, Indexikalität, soziale Registrierung. Zur diskursiven Konstruktion sprachideologischer Positionen“. Zeitschrift für Diskursforschung 1.3 (2013): 263-287.

Vološinov, Valentin N. Marxismus und Sprachphilosophie. Grundlegende Probleme der soziologischen Methode in der Sprachwissenschaft. Hg. u. mit einer Einl. vers. v. Samuel Weber. Übers. v. Renate Horlemann. Frankfurt/M.: Ullstein, 1975 [zuerst russ.: Marksizm i filosofija jazyka. Leningrad: Priboj, 1929]. 
Robert Leucht, Prof. Dr. phil. habil., ist Professor für Neuere deutsche Literaturwissenschaft an der Université de Lausanne. Seine Forschungsschwerpunkte umfassen u.a. Exilliteraturforschung, Utopieforschung und komparatistische Fragestellungen, darunter Kulturtransfer und Übersetzen.

Jürgen Spitzmüller, Prof. Dr. phil. habil., ist Professor für Angewandte Sprachwissenschaft an der Universität Wien (Institut für Sprachwissenschaft). Seine Forschungsschwerpunkte umfassen u.a. Sprachideologien, soziale Positionierung, Soziolinguistik der Schriftlichkeit und Diskurstheorie. 